\title{
Finite temperature effects on the neutrino decoupling in the early Universe
}

\author{
N. Fornengo* \\ Department of Physics and Astronomy, The Johns Hopkins University, Baltimore, Maryland 21218 \\ and Dipartimento di Fisica Teorica, Università di Torino and Istituto Nazionale di Fisica Nucleare, Sezione di Torino via P. Giuria 1, \\ 10125 Torino, Italy \\ C. W. $\mathrm{Kim}^{\dagger}$ \\ Department of Physics and Astronomy, The Johns Hopkins University, Baltimore, Maryland 21218 \\ and School of Physics, Korean Institute for Advanced Study, Seoul 130-012, Korea \\ J. Song \\ Department of Physics and Astronomy, The Johns Hopkins University, Baltimore, Maryland 21218
}

(Received 13 February 1997)

\begin{abstract}
Leading finite temperature effects on the neutrino decoupling temperature in the early Universe have been studied. We have incorporated modifications of the dispersion relation and the phase space distribution due to the presence of particles in the heat bath at a temperature of around $1 \mathrm{MeV}$. Since both the expansion rate of the Universe and the interaction rate of a neutrino are reduced by finite temperature effects, it is necessary to calculate thermal corrections as precisely as possible in order to find the net effect on the neutrino decoupling temperature. We have performed such a calculation by using finite temperature field theory. It has been shown that the finite temperature effects increase the neutrino decoupling temperature by $4.4 \%$, the largest contribution coming from the modification of the phase space due to the thermal bath. [S0556-2821(97)03120-2]
\end{abstract}

PACS number(s): 11.10.Wx, 95.30.Cq, 95.30.Tg

\section{INTRODUCTION}

The standard hot big bang model appears to be a reliable description of the evolution of the early Universe, one of the most remarkable successes being the prediction of the present abundance of light chemical elements from the primordial nucleosynthesis calculations [1]. The Universe is usually described as a hot, dilute gas of particles in nearly thermodynamical equilibrium [2,3]. During the early epochs, the particle species in the thermal bath underwent departure from the equilibrium one after another: one of the typical departures was that of neutrinos, when the temperature of the Universe was about $1 \mathrm{MeV}$ [2]. The neutrino decoupling has important (indirect) effects on the evolution of the Universe, since it happened at the time close to the neutron-to-proton ratio $(n / p)$ freeze-out temperature $T \simeq 0.7 \mathrm{MeV}$ and to the photon reheating by $e^{+} e^{-}$annihilation $\left(T \leqq m_{e}\right)$. The synthesis of light elements depends sensitively on the $n / p$ freeze-out abundance which is determined by the interplay between the weak interaction rates and the expansion rate of the Universe [4-6]. Both rates are influenced by the neutrino decoupling temperature $[2,4]$. Neutrinos which were decoupled early do not share the entropy transfer with electrons, positrons, and photons in the medium. As a consequence, their temperature $T_{\nu}$ becomes slightly lower than that of the other particles in equilibrium. On the contrary, if neutrinos are not totally decoupled when the entropy transfer begins,

\footnotetext{
*Electronic address: fornengo@jhup.pha.jhu.edu, fornengo@to.infn.it

†Electronic address: kim@eta.pha.jhu.edu

*Electronic address: jhsong@eta.pha.jhu.edu
}

they can share part of the $e^{ \pm}$entropy and their temperature would be higher. A small change $\Delta T_{\nu}$ modifies the statistical distribution of neutrinos, and in turn affects both the weak interaction rates which maintain the equilibrium between neutrons and protons, and the expansion rate of the Universe which is due to the change of the neutrino contribution to the total energy density. The overall effect is to shift the $n / p$ freeze-out temperature and hence the $n / p$ abundance when the nucleosynthesis begins. The effect on the present Helium abundance $Y$ was estimated to be of the order $\Delta Y / Y$ $\simeq-0.1\left(\Delta T_{\nu} / T_{\nu}\right)$ (the change is with respect to the calculation in which neutrinos are not reheated by electrons) [4]. Therefore, a precise knowledge of the neutrino decoupling temperature is desirable to gain a confidence in the estimates of the primordial element abundance.

The standard calculation of the neutrino decoupling temperature is based on the assumption that particles in the thermal bath behave like free particles. The interactions are only responsible for the thermodynamical equilibrium, but do not contribute to the energy density of the Universe. However, particles in the medium feel effective potentials due to the interactions with other particles, which modifies their dispersion relations or introduces effective mass for the particle. In addition to this dynamical effect, the phase space available for the interaction is necessarily modified by the statistical distribution of particles in the medium.

Our purpose is to examine whether or not the finite temperature effects can actually lower the neutrino decoupling temperature, leading to possible changes in the nucleosynthesis prediction of the present abundance of light elements. We explicitly and consistently include in the calculations the thermal effective mass of a photon and an electron, and the thermalized phase space distribution in the cross section in 
the Born approximation. Higher order interactions and radiative corrections to the neutrino interactions are not considered here, since we aim at the leading order corrections by the thermal bath. Another medium effect, which is not considered here, is the absorption or the emission of photons in the bath. This effect turns out to be important in the nucleosynthesis calculations for the reactions which involve three body initial or final state (such as neutron decay and its inverse decay), because the additional photon involved modifies sizably the phase space of the reactions [5]. In the case of two body reactions responsible for the thermal equilibrium of neutrinos, however, this higher order effect is small compared with the corrections calculated in the present paper, the overall magnitude of which will be shown to be of the order of $15 \%$ on the interaction rates, leading to a $4 \%$ shift in the neutrino decoupling temperature.

The plan of the paper is as follows. In Sec. II, we discuss how to include finite temperature effects in the calculation. In particular, we will briefly discuss the calculation of effective mass of a photon and an electron in the framework of finite temperature quantum field theory (FTQFT), with the special attention to the $\mathrm{MeV}$ temperature range which is relevant for the neutrino decoupling. Limitations of the previous calculations in applying to the present problem will also be discussed. In Sec. III, we evaluate finite temperature effects on the expansion rate of the Universe and the interaction rate of a neutrino, and show their effects on the neutrino decoupling temperature.

\section{THERMAL EFFECTS}

In the early Universe where the particles are propagating in a thermal bath, rather than in the vacuum, their dynamics and interactions are modified to some extent. The behavior of particles in a thermal bath is systematically described in the framework of FTQFT [9]. There are two equivalent formulations of FTQFT: the imaginary-time and real-time formalisms. In the present paper, we adopt the real-time formalism where the Feynman rules for all the vertices are identical with those in the vacuum, and the presence of the thermal bath is taken into account by the modification of the treelevel propagators of fermions and bosons as [9]

$$
\begin{aligned}
-i S_{T}(p)= & (\not p+m)\left[\frac{1}{p^{2}-m^{2}+i \epsilon}+2 \pi i \delta\left(p^{2}-m^{2}\right)\right. \\
& \left.\times n_{F}(p \cdot u)\right] \text { for fermions, }
\end{aligned}
$$

and

$$
\begin{aligned}
-i D_{T}^{\mu \nu}(k)= & \left(-g^{\mu \nu}+\alpha \frac{k^{\mu} k^{\nu}}{k^{2}}\right)\left[\frac{1}{k^{2}+i \epsilon}-2 \pi i \delta\left(k^{2}\right)\right. \\
& \left.\times n_{B}(p \cdot u)\right] \text { for photons, }
\end{aligned}
$$

where $\alpha$ is the gauge-fixing parameter. In Eqs. (1) and (2), $u^{\mu}$ is the four-velocity of the medium $\left[u^{\mu}=(1, \overrightarrow{0})\right.$ in the rest frame of the medium] and $n_{F, B}$ are defined as

$$
n_{F, B}(x)=\theta(x) f_{F, B}(x)+\theta(-x) f_{F, B}(-x),
$$

where $\theta(x)$ is the step function and $f_{F, B}$ are, respectively, the Fermi-Dirac (FD) and Bose-Einstein (BE) distribution functions

$$
f_{F, B}(x)=\left[\exp \left(\frac{x-\eta}{T}\right) \pm 1\right]^{-1}
$$

In Eq. (4) the $(+)$ and $(-)$ signs refer to fermions and bosons, respectively, and $\eta$ is the chemical potential. In this paper $\eta=0$ is assumed for all the species.

Corrections analogous to that of Eq. (2) should also be applied to the propagators of massive gauge bosons, which are exchanged in the weak interactions of neutrinos. Due to the presence of statistical distribution functions, however, finite temperature corrections to their vacuum propagators are exponentially suppressed at $T \sim 1 \mathrm{MeV} \ll M_{W}, M_{Z}$. This reflects the fact that the bath is too cold to excite those very massive degrees of freedom.

In Eq. (4), $T$ is the temperature of the heat bath measured in the rest frame of the fluid. The presence of the bath does not violate the Lorentz invariance of the system since appropriate definitions of temperature, as well as of all the thermodynamical variables, can be obtained in any reference frame by suitable transformation laws $[10,11]$. Since the early Universe as a thermal bath is conveniently described in the rest frame of the fluid itself, i.e., in the comoving reference frame, temperature $T$ has a direct and simple meaning. We will therefore adopt the comoving frame throughout the paper.

In the FTQFT formalism, the effect of the bath on the dynamical evolution of particles is taken into account by modifications to their dispersion relations, which can be recast in the definition of effective mass for the particle. (This effect can be evaluated by calculating the self-energy of the particle in the heat bath, and will be briefly reviewed in Secs. II A and II B.) The change of mass of the particle modifies its contribution to the energy density of the Universe and therefore its expansion rate. At the same time, it modifies the interaction rate due to the change of dispersion relations in the cross sections and the distribution functions $f(E)$.

In addition, the phase space distribution is also influenced by the background: the presence of the same particles in the surrounding medium as those produced in the interaction processes reduces (enhances) the production probability for fermions (bosons), respectively, according to the statistics of the particles. The final state density factors are modified as follows:

$$
\begin{aligned}
& \frac{d^{3} p^{\prime}}{(2 \pi)^{3} 2 E^{\prime}} \rightarrow \frac{d^{3} p^{\prime}}{(2 \pi)^{3} 2 E^{\prime}}\left[1-f_{F}\left(E^{\prime}\right)\right] \text { for fermions } \\
& \frac{d^{3} k^{\prime}}{(2 \pi)^{3} 2 \omega^{\prime}} \rightarrow \frac{d^{3} k^{\prime}}{(2 \pi)^{3} 2 \omega^{\prime}}\left[1+f_{B}\left(\omega^{\prime}\right)\right] \text { for bosons. }
\end{aligned}
$$

In summary, the influence of the medium on the particle evolution in the Universe is due to the temperaturedependent shifts in the dynamical mass of the particles and the temperature-dependent modification of the interactions between particles. Both modifications will be included in the calculation of the neutrino decoupling temperature in Sec. 
III. We now turn to the discussion of thermal mass of the photon, electron, and neutrino at $T \sim \mathrm{MeV}$.

\section{A. Effective mass of the photon}

In the thermal bath at $T \sim \mathrm{MeV}$, a photon propagates through a medium made of electrons, positrons, and neutrinos. Its propagation is therefore influenced by the interactions with the $e^{+}$and $e^{-}$. The effect of these interactions on the dynamical evolution of the photon is taken into account by calculating the self-energy of the photon in the $e^{ \pm}$background. The one-loop self energy diagram gives

$$
\Pi^{\mu \nu}(k)=\Pi_{0}^{\mu \nu}(k)+\Pi_{T}^{\mu \nu}(k),
$$

where $k^{\mu}$ is the photon four momentum, $\Pi_{0}^{\mu \nu}(k)$ is the vacuum polarization tensor at $T=0$ and

$$
\begin{aligned}
\Pi_{T}^{\mu \nu}= & -2 \pi e^{2} \int \frac{d^{4} p}{(2 \pi)^{4}} \operatorname{Tr}\left[\gamma^{\mu}\left(\not p+k+m_{0}\right) \gamma^{\nu}\left(\not p+m_{0}\right)\right] \\
& \times\left[\frac{\delta\left(p^{2}-m_{0}^{2}\right) f_{F}\left(p^{0}\right)}{(p+k)^{2}-m_{0}^{2}}\right. \\
& \left.+\frac{\delta\left[(p+k)^{2}-m_{0}^{2}\right] f_{F}\left(p^{0}+k^{0}\right)}{p^{2}-m_{0}^{2}}\right]
\end{aligned}
$$

describes finite temperature corrections. In Eq. (8), $p^{\mu}$ is the four-momentum of the electron in the loop and $m_{0}$ denotes the electron mass in the vacuum. The separation of the selfenergy into two parts, one referring to the $T=0$ case and the other coming from the presence of the medium, is attributed to the separation of the electron propagator in Eq. (1). The function $\Pi_{0}^{\mu \nu}(k)$ is divergent and, as usual, is subject to the electric charge renormalization. The function $\Pi_{T}^{\mu \nu}(k)$ induces a finite shift in the photon propagator, generating effective mass for the photon. The vacuum polarization tensor can be decomposed as $[10,12]$

$$
\Pi^{\mu \nu}=\pi_{T}(\bar{k}, w) P^{\mu \nu}+\pi_{L}(\bar{k}, w) Q^{\mu \nu},
$$

where $P^{\mu \nu}$ and $Q^{\mu \nu}$ are orthogonal projection operators (for their explicit form, see [10]) and $\pi_{T}$ and $\pi_{L}$ are scalar functions given by

$$
\begin{gathered}
\pi_{L}(\bar{k}, w)=-\frac{k^{2}}{\overline{k^{2}}} u^{\mu} u^{\nu} \Pi_{\mu \nu}, \\
\pi_{T}(\bar{k}, w)=-\frac{1}{2} \pi_{L}(\bar{k}, w)+\frac{1}{2} g^{\mu \nu} \Pi_{\mu \nu} .
\end{gathered}
$$

In Eqs. (9) and (10), $w=k^{\mu} u_{\mu}$ and $\bar{k}=\sqrt{w^{2}-k^{\mu} k_{\mu}}$. In the comoving frame, $w\left(=k^{0}\right)$ is the energy of the photon and $\bar{k}(=|\vec{k}|)$ denotes the magnitude of its three-momentum. The decomposition in Eq. (9) allows one to write the propagator as (in the Feynman gauge)

$$
\Delta^{\mu \nu}=-\frac{P^{\mu \nu}}{k^{2}-\pi_{T}}-\frac{Q^{\mu \nu}}{k^{2}-\pi_{L}} .
$$

In the comoving frame, the $P^{\mu \nu}$ term describes the transverse modes of the photon field, while the $Q^{\mu \nu}$ term is a linear combination of longitudinal and timelike modes. Equation (11) shows that the propagator of physical (transverse) photon modes has a pole at $k^{2}=\pi_{T}$. This is interpreted as a thermal-generated dynamical mass. The pole $k^{2}=\pi_{L}$ in the nontransverse part of the propagator describes the Debye screening length of the photon in the $e^{ \pm}$plasma $[10,13]$.

Since we are interested in the leading order $O(\alpha)$ correction to the mass of the photon, the vacuum dispersion relations $k^{2}=0$ for the photon and $p^{2}=m_{0}^{2}$ for the electron can be used in the right-hand sides in Eqs. (9) and (10). Therefore, the effective mass of the photon is

$\left[m_{\gamma}^{\mathrm{eff}}(T)\right]^{2}=\operatorname{Re}\left[\pi_{T}(\bar{k}, w)\right]=\frac{8 \alpha}{\pi} T^{2} h\left(\mu_{0}\right)+O\left(\alpha^{2}\right)$,

where $\mu_{0}=m_{0} / T$ and the function $h\left(\mu_{0}\right)$ is defined as

$$
h\left(\mu_{0}\right)=\int_{0}^{\infty} d x \frac{x^{2}}{\sqrt{x^{2}+\left(\mu_{0}\right)^{2}}} f_{F}(x) .
$$

Note that the photon effective mass in Eq. (12) has been obtained without any restriction to $T$, hence valid for all temperature. Equation (12) gives the correct limit $m_{\gamma}=0$ for $T=0$ and is in agreement with the result $\left[m_{\gamma}^{\text {eff }}(T)\right]$ $=\left(2 \pi \alpha T^{2}\right) / 3$ obtained in Ref. [10] in the limit of high temperature $T \gg w, m_{0}$. Obviously this limit does not apply to the early Universe where photons are in thermal equilibrium with the mean energy $w \sim T$. Moreover, since $T \sim m_{0}$ at the neutrino decoupling temperature, the limit $\mu_{0} / x=m_{0} / T \ll 1$ in Ref. [13] cannot be applied.

The thermal mass of the photon is almost linearly dependent on temperature. The photon effective mass is 0.115 $\mathrm{MeV}$ at $T=1 \mathrm{MeV}$ and $0.241 \mathrm{MeV}$ at $T=2 \mathrm{MeV}$. Even though the photon is still relativistic, its contribution to the energy density of the Universe is substantially reduced.

\section{B. Effective mass of the electron}

As in the case of the photon, the dynamics of electrons in a thermal bath is also modified by the electromagnetic interactions with background photons and electrons themselves. The interactions with neutrinos are suppressed at $T \sim 1 \mathrm{MeV}$, for they involve the exchange of heavy bosons $W$ and $Z$. Therefore, the effect of the bath on the propagation of the electron is expressed by calculating the electron self-energy in the presence of the ambient $e^{+}, e^{-}$, and $\gamma$. The electron self-energy at one-loop level becomes

$$
\Sigma(\not p)=\Sigma_{0}(\not p)+\Sigma_{T}(\not p),
$$

where $\Sigma_{0}(\not p)$ is the electron self-energy for $T=0$ and its thermal correction is

$$
\begin{aligned}
\Sigma_{T}(\not p)= & -2 \pi e^{2} \int \frac{d^{4} k}{(2 \pi)^{4}}\left[\gamma^{\mu}\left(\not p+k+m_{0}\right) \gamma_{\mu}\right] \\
& \times\left[\frac{\delta\left(k^{2}\right) f_{B}\left(k^{0}\right)}{(p+k)^{2}-m_{0}^{2}}-\frac{\delta\left[(p+k)^{2}-m_{0}^{2}\right] f_{B}\left(p^{0}+k^{0}\right)}{k^{2}}\right],
\end{aligned}
$$


where $p^{\mu}$ is the four-momentum of the electron and $k^{\mu}$ denotes the momentum in the electron-photon loop. The oneloop self-energy modifies the electron propagator $S(p)$ as

$$
S(p)^{-1}=\not p-m_{0}-\Sigma(\not p)=\left[\not p-m_{0}-\Sigma_{0}(\not p)\right]-\Sigma_{T}(\not p) .
$$

The standard $\Sigma_{0}(\not p)$ leads to the definition of physical mass of electrons and the wave function renormalization in the vacuum. The temperature-dependent self-energy $\Sigma_{T}(\not p)$ produces a finite shift in the dispersion relation as [7]

$$
\begin{aligned}
{\left[m_{e}^{\mathrm{eff}}(T, p)\right]^{2} \equiv } & E^{2}-\vec{p}^{2}=m_{0}^{2}+\frac{2}{3} \alpha \pi T^{2}+\frac{4 \alpha^{2}}{\pi} h\left(\mu_{0}\right) \\
& +\frac{\alpha}{2 \pi^{2}} m_{0}^{2} J_{A}(p)
\end{aligned}
$$

where the function $h\left(\mu_{0}\right)$ is defined in Eq. (13) and

$$
\begin{aligned}
J_{A}(p)= & -\frac{2 \pi}{u} \int_{0}^{\infty} \frac{x d x}{\sqrt{x^{2}+\left(\mu_{0}\right)^{2}}} f_{F}\left(\epsilon_{x}\right) \\
& \times \ln \left(\frac{\epsilon_{p} \epsilon_{x}+\mu_{0}^{2}+x u}{\epsilon_{p} \epsilon_{x}+\mu_{0}^{2}-x u} \frac{\epsilon_{p} \epsilon_{x}-\mu_{0}^{2}+x u}{\epsilon_{p} \epsilon_{x}-\mu_{0}^{2}-x u}\right),
\end{aligned}
$$

where dimensionless quantities have been defined as $u=|\vec{p}| / T, \quad \epsilon_{p}=E_{p} / T, \quad x=|\vec{p}-\vec{k}| / T$, and $\epsilon_{x}=\left(E_{p}-E_{k}\right) / T$. The result of Eq. (17) is valid for all temperature. It gives the correct result $m_{e}^{\text {eff }}=m_{0}$ at $T=0$ and agrees with the result of $[7,14,15]$ in the limiting cases $T \ll m_{0}$ and $T \gg m_{0}$. The last two terms in Eq. (17) are relatively small at $T \ll m_{0}$, for they are exponentially suppressed by the Fermi-Dirac or the Bose-Einstein distribution function. Around $T \sim m_{0}$, however, the third term becomes important and has to be taken into account [for instance, $h\left(m_{0} / T=1\right) \simeq 0.543$ ]. The last term $J_{A}(p)$ in Eq. (17) introduces momentum dependence in the electron effective mass, which is important especially in the calculation of the neutrino cross sections with the momentum-dependent phase space distributions. This term has been calculated in the limit $\epsilon_{x} \gg \mu_{0}$ [14]. This approximation is only valid at high temperature $\left(T \gg m_{0}\right)$, for the most significant contribution of $\epsilon_{x}$ to the integral is from its mean values, i.e., $\epsilon_{x} \sim 1$. In the temperature range of our interest $\left(T \sim m_{0}\right)$, this approximation cannot be adopted and we have to resort to a numerical calculation. This function is always negative, and monotonically increasing from the limiting value

$\lim _{p \rightarrow 0} J_{A}(p)=-8 \pi \int_{0}^{\infty} \frac{d x}{\sqrt{x^{2}+\left(\mu_{0}\right)^{2}}} f_{F}\left(\sqrt{x^{2}+\mu_{0}^{2}}\right)$,

up to $\lim _{p \rightarrow \infty} J_{A}(p)=0$. In order to see how significant the modifications to $m_{0}$ due to the $J_{A}(p)$ term is, we plot in Fig. 1 the deviation of the $m_{e}^{\text {eff }}$ calculation including the $J_{A}(p)$ term from that neglecting $J_{A}(p)$, as a function of temperature. The dashed line refers to the calculation for the case with $p=\langle p\rangle$, where $\langle p\rangle$ is the mean value of the momentum in the bath. The solid line referring to the case with $p=0$ corresponds to the largest contribution of the $J_{A}(p)$ term, the maximum of which is $3.3 \times 10^{-3}$ at $T \sim 2 \mathrm{MeV}$. The other thermal corrections in Eq. (17) are always larger than that due to the $J_{A}(p)$ term at least by one order of magnitude, as can be seen by comparing Fig. 1 to Fig. 2 (in Fig. 2, the relative correction $\left|1-m_{e}^{\mathrm{eff}}(T) / m_{0}\right|$ is plotted as a function of $T)$. This shows that the $J_{A}(p)$ term is negligible around the temperature $T \sim \mathrm{MeV}$. We will therefore neglect this term in our analysis. This turns out to be a great simplification in the calculations, especially for the interaction rate, because the momentum dependence in the electron mass is avoided.

Figure 2 shows that the thermal corrections to the electron mass at $T \sim \mathrm{MeV}$ is sizable: at $T=1 \mathrm{MeV}$ the electron mass increases by $4.1 \%$, and at $T=2 \mathrm{MeV}$ the correction is as large as $16 \%$.

\section{Effective mass of the neutrino}

Neutrinos also acquire effective mass in the presence of a medium. In the temperature range of our interest, the contri-

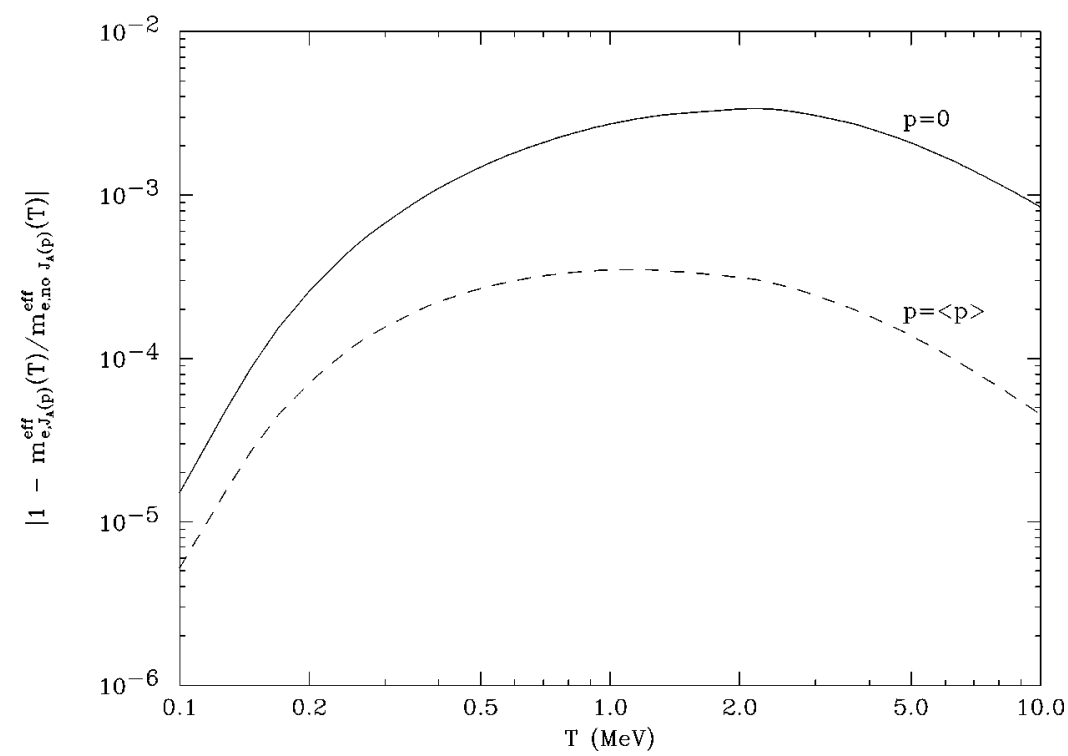

FIG. 1. The deviation of the effective mass of the electron $m_{e}^{\text {eff }}$ calculated by including the $J_{A}(p)$ term from the one without the $J_{A}(p)$ term as a function of $T$. The solid line refers to the electron momentum $p=0$, the dashed line to the mean value $p=\langle p\rangle$. 


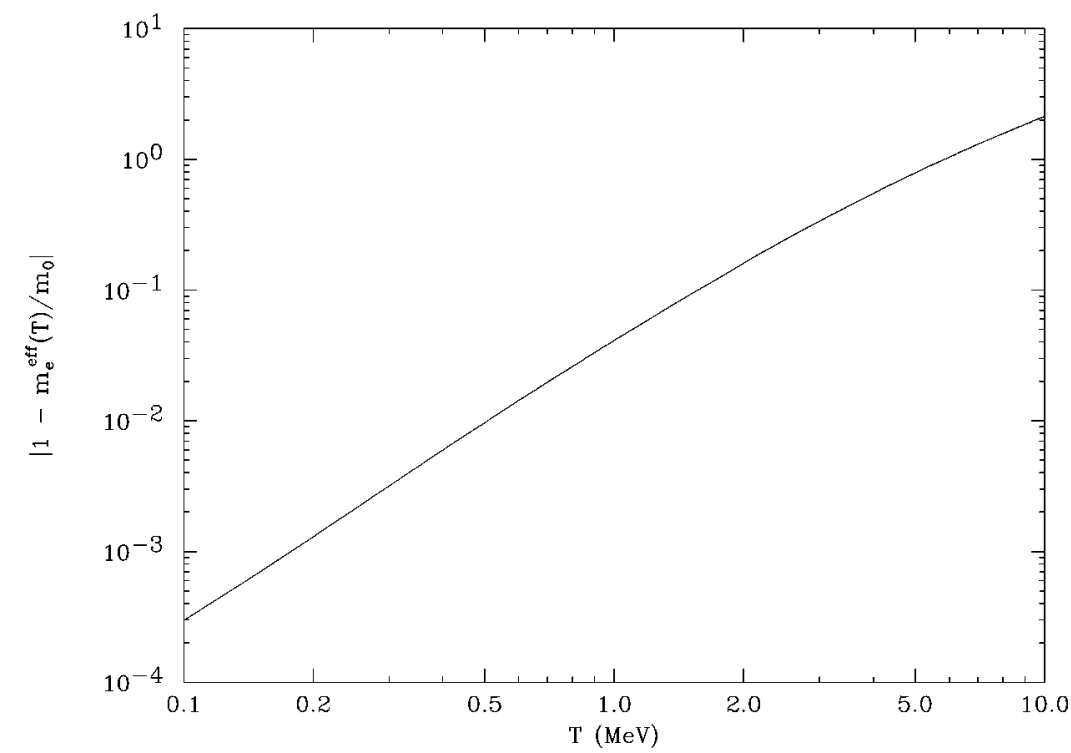

FIG. 2. The deviation of the thermal electron mass $m_{e}^{\text {eff }}(T)$ from $m_{0}$ as a function of $T$.

butions come from weak interactions with the ambient electrons and positrons. The effective mass squared of the neutrino has been shown to be of the order of $G_{F}(N-\bar{N})$, where $G_{F}$ is the Fermi constant and $N(\bar{N})$ is the number density of electrons(positrons) [16]. Since we expect $N \simeq \bar{N}$, the modification to the neutrino effective mass at $T \sim 1 \mathrm{MeV}$ is practically absent. Therefore, we neglect finite temperature effects on the dispersion relation of neutrinos in the following.

\section{NEUTRINO DECOUPLING}

Neutrinos in the early Universe, like all other particles, are kept in thermodynamical equilibrium through their interactions with the particles in the heat bath. As long as its interaction rate $\Gamma$ is larger than the expansion rate of the Universe $H$, neutrinos remain in thermal and chemical equilibrium. When $\Gamma$ becomes smaller than $H$, due to the reduced temperature of the heat bath and therefore the increased distance between particles, neutrinos start to depart from the equilibrium and subsequently evolve independently from the other species. Even though this decoupling is not a sharp event, we can define that it happens when

$$
\Gamma\left(T_{d}\right)=H\left(T_{d}\right)
$$

The temperature when Eq. (20) holds is defined as the neutrino decoupling temperature $T_{d}$. In the following subsections we will discuss finite temperature effects on the calculation of $T_{d}$. Both $\Gamma$ and $H$ decrease as finite temperature effects are incorporated. A detailed calculation is therefore needed to determine whether or not the decoupling temperature actually increases or decreases, and to estimate the size of the effect.

\section{A. Expansion rate}

In the standard big bang model, the dynamical expansion of the early Universe is governed by the Friedmann equation [2]

$$
H=\left[\frac{8 \pi G}{3} \rho\right]^{1 / 2}
$$

where $G$ is the Newton constant. Under the assumption that all the particles are in thermodynamical equilibrium, the total energy density $\rho$ is

$$
\rho=\sum_{i} g_{i} \int \frac{d^{3} p}{2 \pi^{3}} E(p) f_{i}(E)
$$

where $g_{i}$ is the number of internal degrees of freedom of the particle species $i$. $E$ and $p$ are its energy and momentum, respectively, which are related by the usual dispersion relation $E^{2}=\vec{p}^{2}+m_{i}^{2}$, where $m_{i}$ is the mass of the particle.

Equation (22) leads to the following expression of $H$ :

$$
H=\sqrt{\frac{4 \pi^{3} G}{45}} g_{*}^{1 / 2}(T) T^{2}
$$

where $g_{*}(T)$ is the number of degrees of freedom at temperature $T$, defined by

$$
g_{*}(T)=\sum_{i}\left(\frac{T_{i}}{T}\right)^{4} \frac{15 g_{i}}{\pi^{4}} \int_{0}^{\infty} d u \frac{u^{2} \sqrt{u^{2}+\mu_{i}^{2}}}{\exp \left(\sqrt{u^{2}+\mu_{i}^{2}}\right) \pm 1},
$$

where $\mu_{i}=m_{i} / T$. In Eq. (24), $T_{i}$ is the temperature of the species $i$. For the species $i$ in equilibrium, $T_{i}$ is equal to the temperature $T$ of the Universe, but after the decoupling, its temperature does not need to be the same as $T$ (this is actually the case for neutrinos). Note that the dimensionless $g_{*}(T)$ is proportional to the energy density of the Universe in units of $T^{4}$, which is effectively dominated by highly relativistic particles; since particles near the transition from the relativistic to the nonrelativistic regime can also contribute to $g_{*}(T)$, they should be also taken into account in a precise calculation. When a particle species becomes nonrelativistic, its contribution to the energy density and therefore to $g_{*}(T)$ is exponentially suppressed. Around the neutrino decoupling 


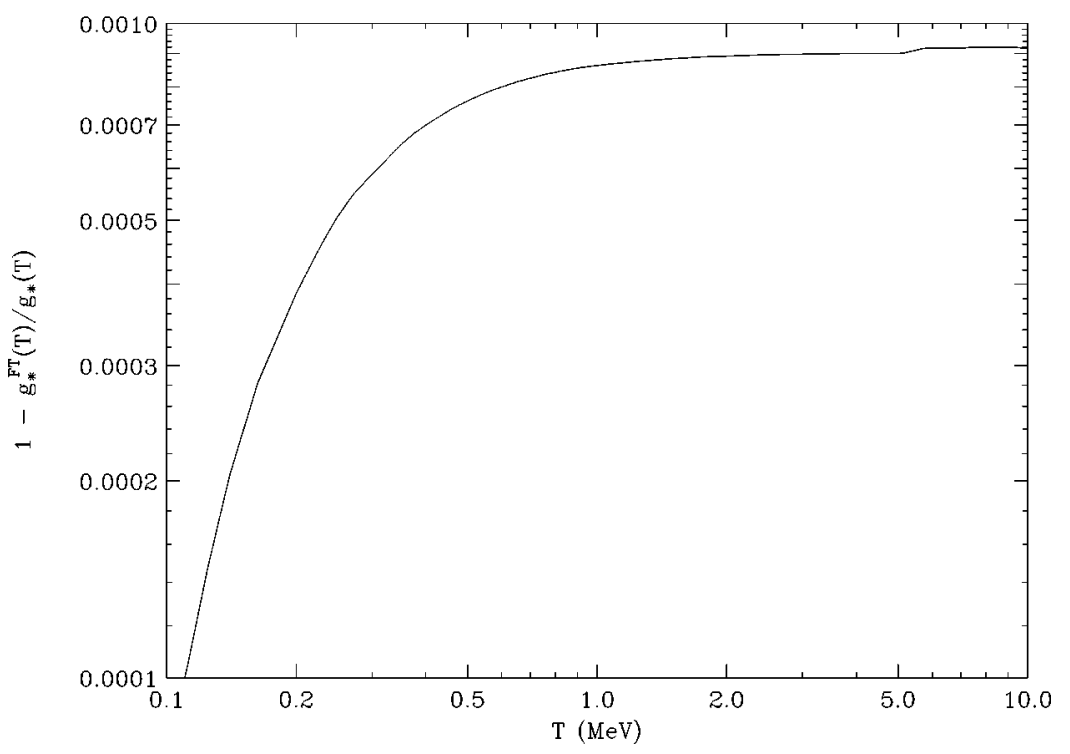

FIG. 3. The deviation of the number of degrees of freedom with finite temperature corrections $g_{*}^{F T}(T)$ from $g_{*}(T)$ as a function of $T$.

temperature $(T \simeq 1 \mathrm{MeV})$, the particles in thermal equilibrium are photons, electrons, positrons, and $\nu_{e}\left(\bar{\nu}_{e}\right)$. We will assume that muon and tau neutrinos had already been decoupled, so that they do not interact with the $\nu_{e}$. However, they still contribute to the energy density.

Now, let us discuss the effects of the thermal background. Since electrons, positrons, and photons have effective mass larger than that in the $T=0$ situation, $g_{*}(T)$ and hence $H(T)$ are reduced. Figure 3 shows the relative change $\left[1-g_{*}^{\mathrm{FT}}(T) / g_{*}(T)\right]$ as a function of temperature. Although the photon mass is a few tenths of a MeV and the electron mass increases by $5 \sim 10 \%$ at the temperatures around $1 \sim 2 \mathrm{MeV}$, the effect on the number of degrees of freedom is small: $g_{*}(T)$ decreases by a factor of $0.1 \%$. This is because in this temperature regime, $g_{*}(T)$ is dominated by three neutrino species. Because of the relation between $H(T)$ and $g_{*}(T)$ in Eq. (23), the ensuing effect on the expansion rate is a factor of two smaller: $\Delta H / H$ $\simeq 0.5\left(\Delta g_{*} / g_{*}\right) \simeq 5 \times 10^{-4}$. As will be shown in Sec. III B, finite temperature modifications to $\Gamma$ are two orders of magnitude larger than that to the expansion rate, leaving very little influence of $H$ on the calculation of $T_{d}$.

The effect of the different assumptions in the calculation of $H$ considered in this paper, are summarized in Table I.

\section{B. Interaction rate and neutrino decoupling temperature}

Neutrinos are kept in thermal equilibrium by the interactions with electrons and positrons in the heat bath. Focusing on the electron neutrino $\nu_{e}$, the interactions such as

TABLE I. The expansion rate $H$ at $T=1.6 \mathrm{MeV}$ under several assumptions. Deviations (in percentage) with respect to the calculation of column (a) are given.

\begin{tabular}{cccc}
\hline \hline (a) & (b) & (c) \\
\hline & $m_{e}=m_{0}$ & $m_{e}=0$ & $m_{e}=m_{e}^{\text {eff }}(T)$ \\
$m_{\gamma}=0$ & $m_{\gamma}=0$ & $m_{\gamma}=m_{\gamma}^{\text {eff }}(T)$ \\
& & & \\
$H /\left(10^{-21} \mathrm{MeV}\right)$ & 1.1396 & 1.1410 & 1.1391 \\
& & $+0.12 \%$ & $-0.045 \%$ \\
\hline \hline
\end{tabular}

$$
\begin{aligned}
& \nu_{e}+e^{-} \leftrightarrow \nu_{e}+e^{-}, \\
& \nu_{e}+e^{+} \leftrightarrow \nu_{e}+e^{+},
\end{aligned}
$$

are responsible for kinetic equilibrium, and annihilation and creation processes like

$$
\nu_{e}+\bar{\nu}_{e} \leftrightarrow e^{-}+e^{+}
$$

maintain neutrinos in chemical equilibrium. Since the interactions which involve more than two particles are suppressed by additional powers of the small coupling constants, we will not consider them in the following. For definiteness, we will discuss in detail the interaction rate for the process of Eq. (25), which has the largest cross section and therefore is dominant in the determination of the thermal equilibrium for $\nu_{e}$. We will turn to the other processes at the end of this section.

The standard calculation of the interaction rate of a neutrino relies on a number of simplifying assumptions: (1) the electron is considered to be massless, i.e., $m_{e}=0$; (2) the energy distribution of the initial-state particles is neglected: two initial particles are considered to have mean-valued energies

$$
\begin{aligned}
& \langle E\rangle=\frac{g_{e}}{N_{e}(T)} \int \frac{d^{3} p}{(2 \pi)^{3}} E f_{e}(E), \\
& \langle\omega\rangle=\frac{g_{\nu}}{N_{\nu}(T)} \int \frac{d^{3} k}{(2 \pi)^{3}} \omega f_{\nu}(\omega),
\end{aligned}
$$

and the interaction is supposed to occur in the center of momentum frame $(\cos \theta=-1)$. Under these assumptions, the interaction rate becomes

$$
\Gamma=N_{e}(T) v_{M} \sigma(\langle E\rangle,\langle\omega\rangle)
$$

where $v_{M}=2$. The cross section $\sigma$ for the massless electron is 


$$
\sigma=\frac{G_{F}^{2} A}{12 \pi} s
$$

where $s=4\langle E\rangle\langle\omega\rangle$ and the definition of the constant $A$ will be given later [see Eq. (47)]. Since the electron is assumed to be massless, we have

$$
\langle E\rangle=\langle\omega\rangle=\frac{7 \pi^{4}}{180 \zeta(3)} T \simeq 3.15 T
$$

and

$$
N_{e}(T)=\frac{3}{4} \frac{\zeta(3)}{\pi^{2}} g_{e} T^{3} \simeq 0.182 T^{3},
$$

where $\zeta$ is the Riemann zeta function and $\zeta(3)=1.20$. Consequently, $\Gamma$ is

$$
\Gamma \simeq 0.385 A G_{F}^{2} T^{5} \simeq 3.48 \times 10^{-22}\left(\frac{T}{\mathrm{MeV}}\right)^{5}
$$

Comparing Eq. (34) with the expansion rate calculated for the massless electron $\left[g_{*}(T)=10.75\right]$,

$$
H \simeq 4.46 \times 10^{-22}\left(\frac{T}{\mathrm{MeV}}\right)^{2},
$$

the decoupling temperature is

$$
T_{d} \simeq 1.09 \mathrm{MeV}
$$

The above standard analysis provides very rough estimates of the interaction rate and the decoupling temperature. For comparison, the calculation of the expansion rate for $m_{e}=0$, but taking into account the thermal distribution of the initial energies, gives

$$
\Gamma \simeq 0.1284 A G_{F}^{2} T^{5}
$$

which is a factor of 3 smaller than the estimate in Eq. (34). The resulting decoupling temperature is

$$
T_{d} \simeq 1.56 \mathrm{MeV},
$$

which is $50 \%$ higher than the value given in Eq. (36).

A detailed and precise calculation of the interaction rate is rather involved, but necessary to see whether or not finite temperature effects lower the neutrino decoupling temperature. For the following interaction

$$
\nu_{e}(\omega, \vec{k})+e^{-}(E, \vec{p}) \leftrightarrow \nu_{e}\left(\omega^{\prime}, \vec{k}^{\prime}\right)+e^{-}\left(E^{\prime}, \vec{p}^{\prime}\right),
$$

the interaction rate is defined by

$$
\begin{aligned}
\Gamma\left(\nu_{e} e^{-}\right. & \left.\rightarrow \nu_{e} e^{-}\right) \\
& =\frac{1}{N_{\nu}(T)} \int \frac{d^{3} p}{(2 \pi)^{3}} \frac{d^{3} k}{(2 \pi)^{3}} g_{e} f_{e}(E) g_{\nu} f_{\nu}(\omega)\left[\sigma v_{M}\right],
\end{aligned}
$$

where the number density $N_{\nu}(T)$ of neutrinos at temperature $T$ is

$$
N_{\nu}(T)=g_{\nu} \int \frac{d^{3} k}{(2 \pi)^{3}} f_{\nu}(\omega) .
$$

That is, $\Gamma$ is the thermal average of product of the cross section $\sigma$ and the Möller velocity $v_{M}$ [17]. The cross section of the process is, including the thermal phase space,

$$
\begin{aligned}
\sigma= & \frac{1}{4 E \omega v_{M}} \int \frac{d^{3} p^{\prime}}{(2 \pi)^{3} 2 E^{\prime}} \frac{d^{3} k^{\prime}}{(2 \pi)^{3} 2 \omega^{\prime}}\left[1-f_{e}\left(E^{\prime}\right)\right] \\
& \times\left[1-f_{\nu}\left(\omega^{\prime}\right)\right](2 \pi)^{4} \delta^{(4)}\left(p+k-p^{\prime}-k^{\prime}\right)|\mathcal{M}|^{2},
\end{aligned}
$$

and the Möller velocity is defined (for massless neutrinos) by

$$
v_{M}=\frac{p^{\alpha} k_{\alpha}}{E \omega}=\frac{s}{2 E \omega}\left(1-\frac{m_{e}^{2}}{s}\right),
$$

where $s \equiv(p+k)^{\alpha}(p+k)_{\alpha}$ is the total energy in the center of momentum frame of the colliding particles and its expression in the comoving frame is ( $\theta$ is the angle between $\vec{p}$ and $\vec{k}$ )

$$
s=m_{e}^{2}+2 E \omega-2 p k \cos \theta .
$$

The scattering amplitude for the process $\nu_{e} e^{-} \rightarrow \nu_{e} e^{-}$ comes from two diagrams with $Z$ in the $t$-channel and $W$ in the $u$-channel. Since mean energies of interacting particles are of the order of the temperature $T \simeq \operatorname{MeV}\left(\ll M_{W, Z}\right)$, we can express the averaged square amplitude in the low energy limit as

$$
\begin{aligned}
|\mathcal{M}|^{2}= & 16 G_{F}^{2}\left[(v+a)^{2}\left(p^{\alpha} k_{\alpha}\right)\left(p^{\prime \alpha} k_{\alpha}^{\prime}\right)+(v-a)^{2}\left(p^{\prime \alpha} k_{\alpha}\right)\right. \\
& \left.\times\left(p^{\alpha} k_{\alpha}^{\prime}\right)-\left(v^{2}-a^{2}\right)^{2} m_{e}^{2}\left(k^{\alpha} k_{\alpha}^{\prime}\right)\right]
\end{aligned}
$$

where $G_{F}$ is the Fermi constant, $v=g_{V}+1, a=g_{A}+1$, and $g_{V}\left(g_{A}\right)$ are the vector (axial-vector) weak coupling constant.

As a first step, we neglect the thermal phase space in order to investigate the effect of electron thermal mass alone. With the standard phase space, the cross section is

$$
\sigma=\frac{G_{F}^{2}}{12 \pi} s\left(1-\frac{m_{e}^{2}}{s}\right)^{2}\left[A+B \frac{m_{e}^{2}}{s}+C \frac{m_{e}^{4}}{s^{2}}\right],
$$

where

$$
\begin{gathered}
A=4\left(a^{2}+a v+v^{2}\right), \\
B=2\left(2 a^{2}-a v-v^{2}\right), \\
C=(a-v)^{2} .
\end{gathered}
$$

Finally, $\Gamma$ is to be obtained by taking a thermal average of $\sigma v_{M}$. A simple expression of $\Gamma$ in terms of Bessel functions, 


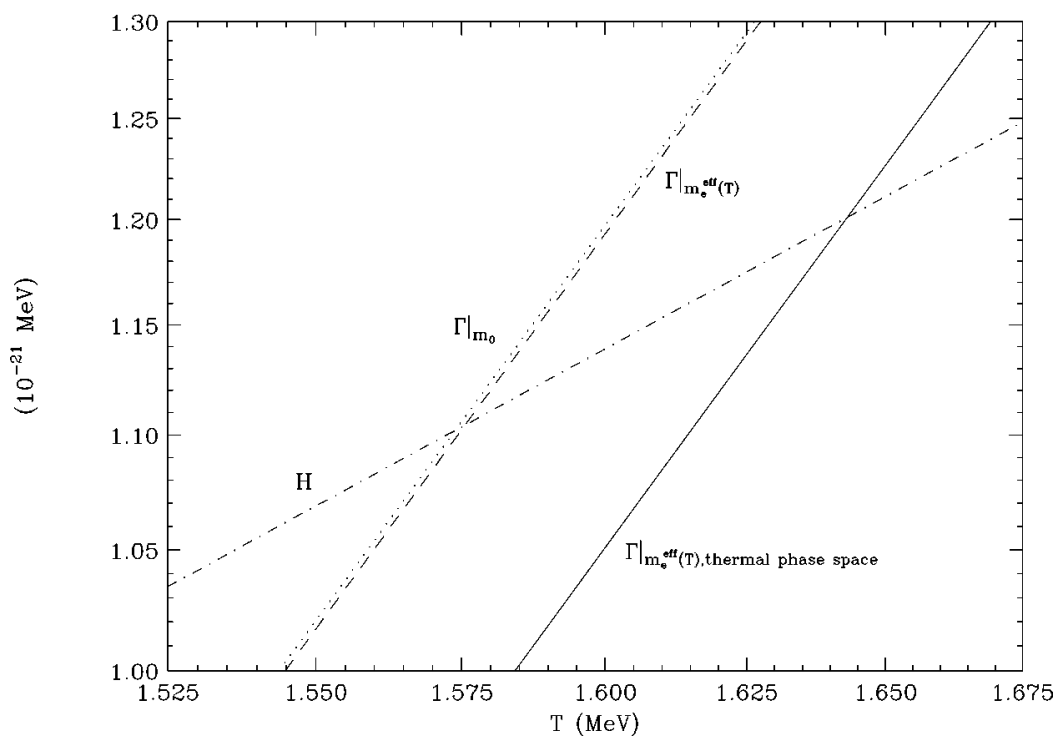

FIG. 4. The expansion rate $H$ (dash-dotted line) and the interaction rate $\Gamma$ for the reaction $\nu_{e}+e^{-} \leftrightarrow \nu_{e}+e^{-}$as functions of $T$, in units of $10^{-21} \mathrm{MeV}$. The dotted line refers to $\Gamma$ when the thermal corrections are neglected. The dashed line represents the case with the electron thermal mass. The solid line includes both the electron thermal mass and the thermal phase space.

involving a one-dimensional numerical integration, has been obtained in the case of Maxwell-Boltzmann (MB) distribution for the initial particles [17]. We do not adopt this approximation here, because it induces larger uncertainties than the finite temperature corrections in the present analysis. For instance, particles with energy $E \sim\langle E\rangle$ would have $4.7 \%$ larger interaction rate in the MB distribution than in the FD distribution. Therefore, we have to resort to an improved method [18].

Since the only angular dependence comes from the relative angle $\theta$ between $\vec{p}$ and $\vec{k}$, we have

$$
d^{3} p d^{3} k=4 \pi p^{2} d p 2 \pi k^{2} d k d \cos \theta
$$

with the kinematical limits $(0, \infty)$ for both $p$ and $k$ and $|\cos \theta| \leqslant 1$. With the isotropic distribution functions, the annihilation rate is

$$
\begin{aligned}
\Gamma= & \frac{1}{N_{\nu}(T)} \frac{g_{e} g_{\nu}}{8 \pi^{4}} \frac{G_{F}^{2}}{12 \pi} \int_{0}^{\infty} \frac{p^{2} d p}{E} f_{e}(E) \\
& \times \int_{0}^{\infty} \frac{k^{2} d k}{\omega} f_{\nu}(\omega) I(p, k),
\end{aligned}
$$

where

$$
\begin{aligned}
I(p, k) & =\frac{1}{2} \int_{-1}^{1} d \cos \theta s^{2}\left(1-\frac{m_{e}^{2}}{s}\right)^{3}\left[A+B \frac{m_{e}^{2}}{s}+C \frac{m_{e}^{4}}{s^{2}}\right] \\
& =\frac{1}{2} \sum_{i=1}^{6} a_{i} I_{i},
\end{aligned}
$$

where the constants $a_{i}$ and the functions $I_{i}$ are obtained from the angular integration as

$$
\begin{gathered}
a_{1}=m_{e}^{4}(C+3 A-3 B), \\
a_{2}=m_{e}^{2}(B-3 A), \\
a_{3}=A, \\
a_{4}=m_{e}^{6}(3 B-A-3 C), \\
a_{5}=m_{e}^{8}(3 C-B), \\
a_{6}=-m_{e}^{10} C,
\end{gathered}
$$

and

$$
\begin{aligned}
& I_{1}=2, \\
& I_{2}=2\left(m_{e}^{2}+2 E \omega\right), \\
& I_{3}=2\left(m_{e}^{2}+2 E \omega\right)^{2}+\frac{8}{3} p^{2} \omega^{2} \\
& I_{4}=\frac{1}{2 p \omega} \ln \left[\frac{m_{e}^{2}+2 E \omega+2 p \omega}{m_{e}^{2}+2 E \omega-2 p \omega}\right], \\
& I_{5}=\frac{1}{2 p \omega}\left[\frac{1}{m_{e}^{2}+2 E \omega-2 p \omega}-\frac{1}{m_{e}^{2}+2 E \omega+2 p \omega}\right], \\
& I_{6}=\frac{1}{4 p \omega}\left[\frac{1}{\left(m_{e}^{2}+2 E \omega-2 p \omega\right)^{2}}-\frac{1}{\left(m_{e}^{2}+2 E \omega+2 p \omega\right)^{2}}\right] .
\end{aligned}
$$

The above holds for all interaction rates as long as the involved electron has momentum-independent mass. The integrations over $p$ and $k$ in Eq. (51) are performed numerically (all the numerical calculations are performed with a precision of $10^{-5}$ but only three significant decimals are shown).

The interaction rate for $m_{e}=m_{0}$, i.e., without any thermal correction, is plotted in Fig. 4 (dotted line) as a function of 


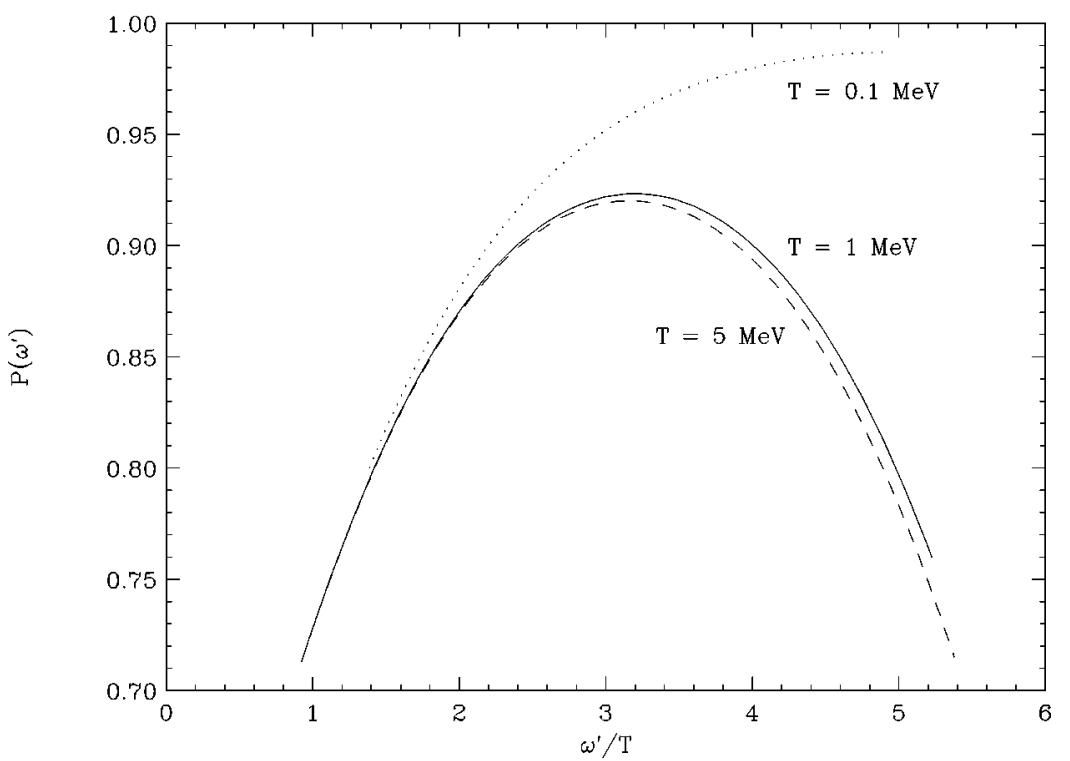

FIG. 5. The phase space reduction function $P\left(\omega^{\prime}\right)$ plotted as a function of the final-state neutrino energy $\omega^{\prime}$ and calculated for the mean values $E=\langle E\rangle$ and $w=\langle w\rangle$ of the initial-state energies with $\cos \theta=0$. The three curves refer to different values of $T: T=0.1 \mathrm{MeV}$ (dotted line), $T=1 \mathrm{MeV}$ (solid line) and $T=5 \mathrm{MeV}$ (dashed line).

temperature. The decoupling condition Eq. (20) is satisfied at the temperature

$$
T_{d}\left(m_{e}=m_{0}\right)=1.57 \mathrm{MeV}
$$

The inclusion of the electron thermal mass has the effect to reduce slightly the interaction rate (see the dashed line in Fig. 4): around $T \simeq 1.6 \mathrm{MeV}$ the reduction is $0.3 \%$. As a consequence, the decoupling temperature increases by $0.6 \%$, i.e.,

$$
T_{d}\left(m_{e}=m_{e}^{\mathrm{eff}}\right)=1.58 \mathrm{MeV}
$$

Let us now take into account the thermal phase space in the evaluation of the cross section, which is

$$
\begin{aligned}
d \Pi= & \frac{d^{3} p^{\prime}}{(2 \pi)^{3} 2 E^{\prime}} \frac{d^{3} k^{\prime}}{(2 \pi)^{3} 2 \omega^{\prime}}\left[1-f_{e}\left(E^{\prime}\right)\right]\left[1-f_{\nu}\left(\omega^{\prime}\right)\right] \\
& \times(2 \pi)^{4} \delta^{(4)}\left(p+k-p^{\prime}-k^{\prime}\right) .
\end{aligned}
$$

The three-momentum $\vec{p}^{\prime}$ can be integrated out by using the three-dimensional delta function to give

$$
\begin{aligned}
d \Pi= & \frac{1}{(2 \pi)^{2} 4 E^{\prime} \omega^{\prime}} k^{\prime 2} d k^{\prime} d \phi_{k^{\prime}} d \cos \theta_{k^{\prime}}\left[1-f_{e}\left(E^{\prime}\right)\right] \\
& \times\left[1-f_{\nu}\left(\omega^{\prime}\right)\right] \delta\left(E+\omega-E^{\prime}-\omega^{\prime}\right)
\end{aligned}
$$

where the angle $\theta_{k^{\prime}}$ is defined as

$$
\cos \theta_{k^{\prime}}=\frac{(\vec{p}+\vec{k}) \cdot \vec{k}^{\prime}}{|\vec{p}+\vec{k}|\left|\vec{k}^{\prime}\right|}
$$

After a trivial integration over $\phi_{k^{\prime}}$, we use the remaining one-dimensional delta function to perform the integration over $\cos \theta_{k^{\prime}}$ :

$$
d \Pi=\frac{1}{8 \pi} \frac{\omega^{\prime}}{E^{\prime}}\left|\frac{\partial\left(E^{\prime}+\omega^{\prime}\right)}{\partial \cos \theta_{k^{\prime}}}\right|^{-1}\left[1-f_{e}\left(E^{\prime}\right)\right]\left[1-f_{\nu}\left(\omega^{\prime}\right)\right] d \omega^{\prime},
$$

with the constraint $\vec{p}+\vec{k}=\vec{p}^{\prime}+\vec{k}^{\prime}$. This gives

$$
d \Pi=\frac{1}{8 \pi \mathcal{A}} P\left(\omega^{\prime}\right) d \omega^{\prime},
$$

where we have defined

$$
P\left(\omega^{\prime}\right)=\left\{1-f_{e}\left[E^{\prime}\left(\omega^{\prime}\right)\right]\right\}\left[1-f_{\nu}\left(\omega^{\prime}\right)\right],
$$

with

$$
\begin{gathered}
E^{\prime}\left(\omega^{\prime}\right)=\alpha-\omega^{\prime}, \\
\alpha=E+\omega \\
\mathcal{A}=|\vec{p}+\vec{k}|=\left(p^{2}+k^{2}+2 p k \cos \theta\right)^{1 / 2} .
\end{gathered}
$$

The quantities $\alpha$ and $\mathcal{A}$ are fixed with the given initial state variables. The kinematical limits for the final state energy $\omega^{\prime}$ are

$$
\begin{aligned}
& \omega_{\max }^{\prime}=\frac{\alpha^{2}-\mathcal{A}^{2}-m_{e}^{2}}{2(\alpha-\mathcal{A})}, \\
& \omega_{\min }^{\prime}=\frac{\alpha^{2}-\mathcal{A}^{2}-m_{e}^{2}}{2(\alpha+\mathcal{A})},
\end{aligned}
$$

which can be obtained from the constraint $\left|\cos \theta_{k^{\prime}}\right| \leqslant 1$.

The relevant phase-space distribution $P\left(\omega^{\prime}\right)$ is a function of the energy $\omega^{\prime}$ and of the energies and momenta of initial particles. In the case of nonthermal phase space, we have $P\left(\omega^{\prime}\right)=1$. The function $P\left(\omega^{\prime}\right)$ represents the reduction of the phase-space due to thermal effects. Figure 5 shows as a function of $\omega^{\prime} P\left(\omega^{\prime}, E=\langle E\rangle, \omega=\langle\omega\rangle\right)$ with $\cos \theta=0$ at 


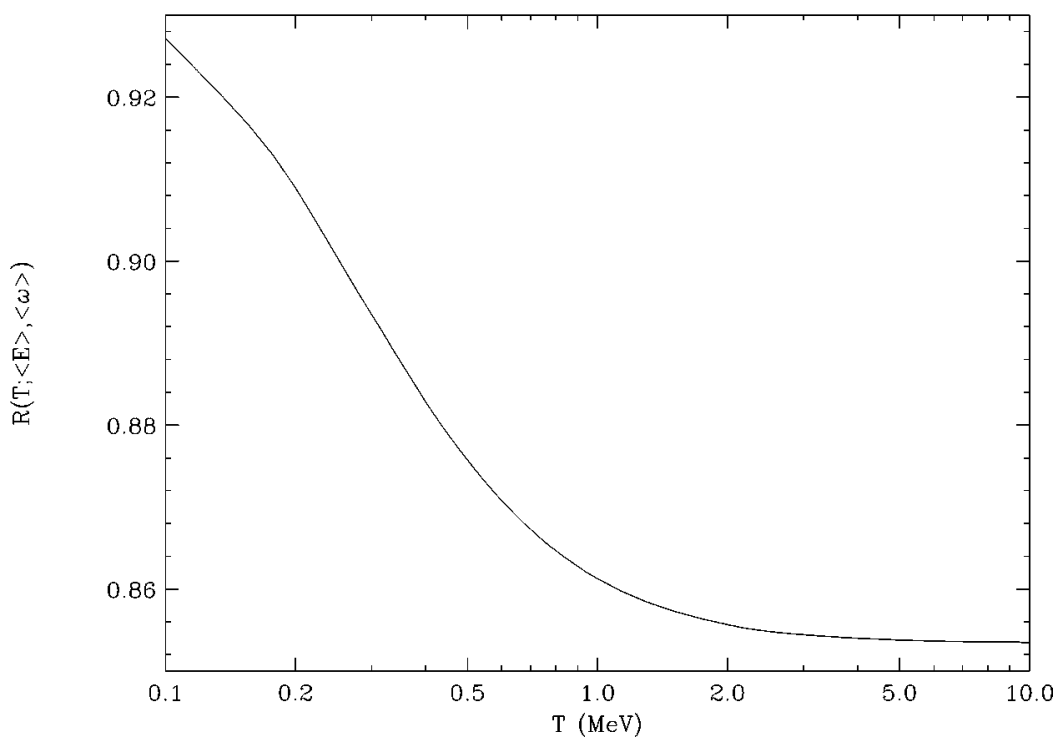

FIG. 6. The thermal phase space reduction factor $R(T ; E, \omega)$ as a function of $T$, calculated for $E=\langle E\rangle$ and $\omega=\langle\omega\rangle$.

three different temperature $T=0.1,1,5 \mathrm{MeV}$. We observe that the reduction due to the thermal distribution function is sizable over all the energy range of $\omega^{\prime}$. Especially around the temperature $T \sim \mathrm{MeV}$, the amount of the reduction is always greater than $8 \%$ for all energies.

Unfortunately, the functional form of $P\left(\omega^{\prime}\right)$ prevents us from analytically performing the integration over the final state energy $\omega^{\prime}$ and the initial state angle $\theta$, contrary to the previous situation without the thermal phase space. In order to minimize numerical integrations and to gain a better understanding of the effect induced by the thermal phase space, we approximate the effect of the thermal phase-space as a mean value effect

$$
\begin{aligned}
4 E \omega\left(\sigma v_{M}\right)_{T H} & =\frac{1}{8 \pi \mathcal{A}} \int P\left(\omega^{\prime}\right)\left|\mathcal{M}^{2}\right| d \omega^{\prime} \\
& \simeq \frac{1}{8 \pi \mathcal{A}}\left\langle P\left(\omega^{\prime}\right)\right\rangle \int\left|\mathcal{M}^{2}\right| d \omega^{\prime} \\
& =4 E \omega\left(\sigma v_{M}\right)_{0}\left\langle P\left(\omega^{\prime}\right)\right\rangle,
\end{aligned}
$$

where the quantity $E \omega\left(\sigma v_{M}\right)_{0}$ is the same as the one calculated previously [see Eqs. (43) and (46)]. In Eq. (66) the mean value of the phase space is defined as

$$
\begin{aligned}
\left\langle P\left(\omega^{\prime}\right)\right\rangle & \equiv R(T ; E, \omega) \\
& =\frac{1}{2} \int_{-n 1}^{1} d \cos \theta \frac{1}{\omega_{\max }^{\prime}-\omega_{\min }^{\prime}} \int_{\omega_{\min }^{\prime}}^{\omega_{\max }^{\prime}} P\left(\omega^{\prime}\right) d \omega^{\prime} .
\end{aligned}
$$

The reduction factor $R(T ; E, \omega)$ is a function of the initial state energies and the temperature. In Fig. $6, R(T ; E, \omega)$ is plotted against the temperature for $E=\langle E\rangle$ and $\omega=\langle\omega\rangle$. As the temperature increases, $R$ decreases because the Fermi blocking at higher temperature obstructs scattering processes. For instance, $R(\langle E\rangle,\langle\omega\rangle)=0.86$ at $T=1 \mathrm{MeV}$. Note that the effect even at $T=0.1 \mathrm{MeV}$ is about $8 \%$ decrease, where the finite temperature effects due to the thermal mass are totally negligible.

With the definition of the reduction factor $R(T ; E, \omega)$, the interaction rate including all the thermal effects is

$$
\begin{aligned}
\Gamma= & \frac{1}{N_{\nu}(T)} \frac{g_{e} g_{\nu}}{8 \pi^{4}} \frac{G_{F}^{2}}{12 \pi} \int_{0}^{\infty} \frac{p^{2} d p}{E} f_{e}(E) \\
& \times \int_{0}^{\infty} \frac{k^{2} d k}{\omega} f_{\nu}(\omega) I(p, k) R(T ; E, \omega) .
\end{aligned}
$$

TABLE II. The interaction rate $\Gamma$ (in units of $10^{-21} \mathrm{MeV}$ ) for the process $\nu_{e} e^{-} \leftrightarrow \nu_{e} e^{-}$at $T=1.6 \mathrm{MeV}$ under several assumptions (ISDF stands for initial-state energy distribution functions; TPS for thermal phase space). Deviations (in percentage) with respect to the calculation of column (a) are given.

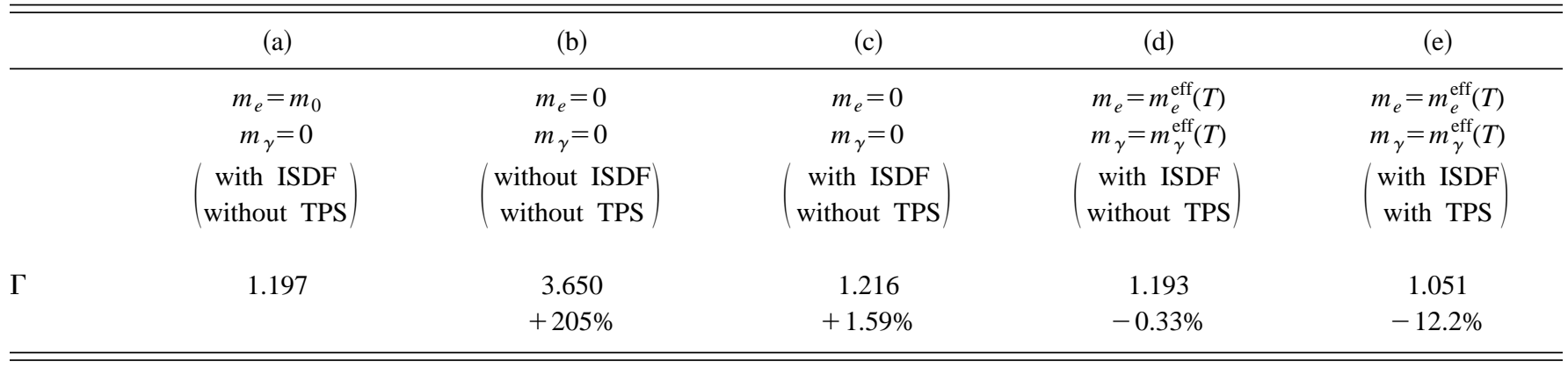


TABLE III. The decoupling temperature $T_{d}$ for the process $\nu_{e} e^{-} \leftrightarrow \nu_{e} e^{-}$under several assumptions. (ISDF stands for initial-state energy distribution functions; TPS for thermal phase space). Deviations (in percentage) with respect to the calculation of column (a) are given.

\begin{tabular}{|c|c|c|c|c|c|}
\hline & (a) & (b) & (c) & (d) & (e) \\
\hline & $\begin{array}{c}m_{e}=m_{0} \\
m_{\gamma}=0 \\
\left(\begin{array}{c}\text { with ISDF } \\
\text { without TPS }\end{array}\right)\end{array}$ & $\begin{array}{c}m_{e}=0 \\
m_{\gamma}=0 \\
\left(\begin{array}{c}\text { without ISDF } \\
\text { without TPS }\end{array}\right)\end{array}$ & $\begin{array}{c}m_{e}=0 \\
m_{\gamma}=0 \\
\left(\begin{array}{c}\text { with ISDF } \\
\text { without TPS }\end{array}\right)\end{array}$ & $\begin{array}{c}m_{e}=m_{e}^{\mathrm{eff}}(T) \\
m_{\gamma}=m_{\gamma}^{\mathrm{eff}}(T) \\
\left(\begin{array}{c}\text { with ISDF } \\
\text { without TPS }\end{array}\right)\end{array}$ & $\begin{array}{c}m_{e}=m_{e}^{\text {eff }}(T) \\
m_{\gamma}=m_{\gamma}^{\text {eff }}(T) \\
\left(\begin{array}{c}\text { with ISDF } \\
\text { with TPS }\end{array}\right)\end{array}$ \\
\hline$T_{d}(\mathrm{MeV})$ & 1.57 & $\begin{array}{c}1.09 \\
-30.6 \%\end{array}$ & $\begin{array}{c}1.56 \\
-0.64 \%\end{array}$ & $\begin{array}{c}1.58 \\
+0.64 \%\end{array}$ & $\begin{array}{c}1.64 \\
+4.4 \%\end{array}$ \\
\hline
\end{tabular}

The interaction rate of Eq. (68) is plotted in Fig. 4 as a solid line. The global reduction of $\Gamma$, compared to the situation without the thermal phase space, is $13 \%$ for $T \sim \mathrm{MeV}$. This is by far the most important effect due to the presence of the thermal bath, being more than a factor of 40 larger than the inclusion of the electron thermal mass alone in the calculation of $\Gamma$. We recall that the thermal mass affects the expansion rate much less, leading to $\Delta H / H \sim 5 \times 10^{-4}$ in the $\mathrm{MeV}$ range of the temperature. The decoupling temperature obtained from $\Gamma$ in Eq. (68) is, finally,

$$
T_{d}\left(m_{e}=m_{e}^{\text {eff }} \text {, thermal phase space }\right)=1.643 \mathrm{MeV},
$$

which is a $4.4 \%$ increase from the decoupling temperature without any thermal effect. We therefore conclude that the thermal bath has the effect to increase the neutrino decoupling temperature, assuring that neutrinos are totally decoupled at the time of $e^{ \pm}$entropy release.

The calculation of the interaction rate $\Gamma\left(\nu_{e} e^{-} \leftrightarrow \nu_{e} e^{-}\right)$ under the different assumptions considered in this paper, are summarized in Table II. The corresponding values of the decoupling temperature $T_{d}\left(\nu_{e} e^{-} \leftrightarrow \nu_{e} e^{-}\right)$are reported in Table III.

For completeness, we conclude this section by reporting the neutrino decoupling temperature taking into account all the reactions listed in Eqs. (25-27). Including the thermal mass and the thermal phase space as discussed above, the neutrino decoupling temperature increases by $4.4 \%$ into

$$
T_{d} \simeq 1.41 \mathrm{MeV},
$$

as compared to $T_{d} \simeq 1.35 \mathrm{MeV}$ obtained without finite temperature effects.

\section{CONCLUSIONS}

We have studied leading finite temperature effects on the neutrino decoupling temperature in the early Universe. The major motivation is to investigate if finite temperature effects could actually lower $T_{d}$ and eventually affect the nucleosynthesis calculations. Two major features of the finite temperature effects have been incorporated in the calculation: (1) the interactions among the particles in the medium affect their dispersion relations which are recast in the form of effective mass; (2) the presence of the medium modifies the phase space distribution of the particles in the processes that determine the equilibrium.

The effect of the inclusion of thermal mass into the expansion rate of the Universe $H$ has been shown very small: $\Delta H / H \simeq 5 \times 10^{-4}$. This effect turns out to be negligible compared to the modification of the interaction rate of a neutrino due to finite temperature. The latter has been discussed in detail for the process $\nu_{e}+e \leftrightarrow \nu_{e}+e$. The incorporation of the electron thermal mass alone leads to a $0.6 \%$ increase of $T_{d}$. When the thermal phase space in the Born approximation is also considered, the decoupling temperature further increases. The total thermal effect is an increase of $T_{d}$ by $4.4 \%$, and the actual value of the decoupling temperature is $T_{d}$ (thermal effects) $=1.41 \mathrm{MeV}$. In conclusion, it is still valid even in the presence of a heat bath that neutrinos are totally decoupled at the time of $e^{ \pm}$entropy transfer.

\section{ACKNOWLEDGMENTS}

N.F. gratefully acknowledges the financial support from the Istituto Nazionale di Fisica Nucleare, Italy.
[1] For an update review on the status of the big bang nucleosynthesis and discussions of the recent controversy about the confrontation of the predictions with the primordial abundances inferred from the latest data, see K. A. Olive, in Neutrinos, Dark Matter and the Universe, Proceedings of the VIII Recontres de Blois, Blois, France, 1996, edited by T. Stolarczyk et al. (Editions Frontieres, Gif-sur-Yvette, 1997), astro-ph/9609071; S. Sarkar, Rep. Prog. Phys. 59, 1493 (1996); G. Steigman, in TAUP 95, Proceedings of the IVth International Workshop on Theoretical and Phenomenological
Aspects of Underground Physics, Toledo, Spain, 1995, edited by A. Morales et al. [Nucl. Phys. B (Proc. Suppl.) 48, 499 (1996)].

[2] S. Weinberg, Gravitation and Cosmology (Wiley, New York, 1972); E. W. Kolb and M. S. Turner, The Early Universe (Addison-Wesley, Reading, MA, 1990).

[3] Nonequilibrium corrections to the energy spectra of particles in an expanding Universe have also been studied. See, for example, A. D. Dolgov, Nucl. Phys. B496, 437 (1997), and references therein. 
[4] D. A. Dicus et al., Phys. Rev. D 26, 2694 (1982).

[5] J. L. Cambier, J. R. Primack, and M. Sher, Nucl. Phys. B209, 372 (1982).

[6] For a detailed discussion about different approximations used to include finite temperature radiative corrections in the nucleosynthesis calculations, see Ref. [7] and Ref. [8].

[7] J. F. Donoghue and B. R. Holstein, Phys. Rev. D 28, 340 (1983); J. F. Donoghue, B. R. Holstein, and R. W. Robinett, Ann. Phys. (N.Y.) 164, 233 (1985).

[8] R. F. Sawyer, Phys. Rev. D 53, 4232 (1996); I. A. Chapman, ibid. 55, 6287 (1997).

[9] C. Bernard, Phys. Rev. D 9, 3312 (1974); L. Dolan and R. Jackiw, ibid. 9, 3320 (1974); S. Weinberg, ibid. 9, 3357 (1974).

[10] H. A. Weldon, Phys. Rev. D 26, 1394 (1982).

[11] R. C. Tolman, Relativity, Thermodynamics and Cosmology
(Clarendon, Oxford, 1934), Chap. 5.

[12] M. B. Kislinger and P. D. Morley, Phys. Rev. D 13, 2765 (1976).

[13] K. Ahmed and S. S. Masood, Ann. Phys. (N.Y.) 207, 460 (1991).

[14] K. Ahmed and S. Saleem, Phys. Rev. D 35, 1861 (1987).

[15] H. A. Weldon, Phys. Rev. D 26, 2789 (1982).

[16] D. Nötzold and G. G. Raffelt, Nucl. Phys. B307, 924 (1988); P. B. Pal and T. N. Pham, Phys. Rev. D 40, 259 (1989); J. F. Nieves, ibid. 40, 866 (1989). See also C. W. Kim and A. Pevsner, Neutrinos in Physics and Astrophysics (Harwood, Academic, Chur, Switzerland, 1993), Chap. 8.

[17] P. Gondolo and G. Gelmini, Nucl. Phys. B360, 145 (1991).

[18] Fermi-Dirac corrections to the evolution of a particle in the early Universe have also been considered in A. D. Dolgov and K. Kainulainen, Nucl. Phys. B402, 349 (1993). 